Network Working Group

Request for Comments: 4042

Category: Informational
M. Crispin

Panda Programming

1 April 2005

\title{
$\mathrm{UTF}-9$ and UTF-18 \\ Efficient Transformation Formats of Unicode
}

Status of This Memo

This memo provides information for the Internet community. It does not specify an Internet standard of any kind. Distribution of this memo is unlimited.

Copyright Notice

Copyright (C) The Internet Society (2005).

Abstract

ISO-10646 defines a large character set called the Universal

Character set (UCS), which encompasses most of the world's writing

systems. The same set of codepoints is defined by Unicode, which

further defines additional character properties and other

implementation details. By policy of the relevant standardization

committees, changes to Unicode and amendments and additions to

ISO/IEC 646 track each other, so that the character repertoires and

code point assignments remain in synchronization.

The current representation formats for Unicode (UTF-7, UTF-8, UTF-16)

are not storage and computation efficient on platforms that utilize

the 9 bit nonet as a natural storage unit instead of the 8 bit octet.

This document describes a transformation format of Unicode that takes advantage of the nonet so that the format will be storage and computation efficient.

1. Introduction

A number of Internet sites utilize platforms that are not based upon the traditional 8-bit byte or octet. One such platform is the PDP10, which is based upon a 36-bit word. On these platforms, it is wasteful to represent data in octets, since 4 bits are left unused in each word. The 9-bit nonet is a much more sensible representation.

Although these platforms support IETF standards, many of these platforms still utilize a text representation based upon the septet, 
which is only suitable for [US-ASCII] (although it has been used for various ISO 10646 national variants).

To maximize international and multi-lingual interoperability, the IAB has recommended ([IAB-CHARACTER]) that [ISO-10646] be the default coded character set.

Although other transformation formats of [UNICODE] exist, and conceivably can be used on nonet-oriented machines (most notably [UTF-8]), they suffer significant disadvantages:

\section{[UTF-8 ]}

requires one to three octets to represent codepoints in the Basic Multilingual Plane (BMP), four octets to represent [UNICODE] codepoints outside the BMP, and six octets to represent non-[UNICODE] codepoints. When stored in nonets, this results in as many as four wasted bits per [UNICODE] character.

\section{[UTF-16]}

requires a hexadecet to represent codepoints in the BMP, and two hexadecets to represent [UNICODE] codepoints outside the BMP. When stored in nonet pairs, this results in as many as four wasted bits per [UNICODE] character. This transformation format requires complex surrogates to represent codepoints outside the BMP, and can not represent non-[UNICODE] codepoints at all.

[UTF-7]

requires one to five septets to represent codepoints in the BMP, and as many as eight septets to represent codepoints outside the BMP. When stored in nonets, this results in as many as sixteen wasted bits per character. This transformation format requires very complex and computationally expensive shifting and "modified BASE64" processing, and can not represent non-[UNICODE] codepoints at all.

By comparison, UTF-9 uses one to two nonets to represent codepoints in the BMP, three nonets to represent [UNICODE] codepoints outside the BMP, and three or four nonets to represent non-[UNICODE] codepoints. There are no wasted bits, and as the examples in this document demonstrate, the computational processing is minimal.

Transformation between [UTF-8] and UTF-9 is straightforward, with most of the complexity in the handling of [UTF-8]. It is hoped that future extensions to protocols such as SMTP will permit the use of UTF-9 in these protocols between nonet platforms without the use of [UTF-8] as an "on the wire" format. 
Similarly, transformation between [UNICODE] codepoints and UTF-18 is also quite simple. Although (like UCS-2) UTF-18 only represents a subset of the available [UNICODE] codepoints, it encompasses the non-private codepoints that are currently assigned in [UNICODE] .

\subsection{Conventions Used in This Document}

The key words "MUST", "MUST NOT", "REQUIRED", "SHALL", "SHALL NOT", "SHOULD", "SHOULD NOT", "RECOMMENDED", "MAY", and "OPTIONAL" in this document are to be interpreted as described in BCP 14, RFC 2119 [KEYWORDS ] .

\section{Overview}

UTF-9 encodes [UNICODE] codepoints in the low order 8 bits of a nonet, using the high order bit to indicate continuation. Surrogates are not used.

[UNICODE] codepoints in the range U+0000 - U+00FF ([US-ASCII] and Latin 1) are represented by a single nonet; codepoints in the range $\mathrm{U}+0100$ - U+FFFF (the remainder of the BMP) are represented by two nonets; and codepoints in the range U+1000 - U+10FFFF (remainder of [UNICODE]) are represented by three nonets.

Non-[UNICODE] codepoints in [ISO-10646] (that is, codepoints in the range 0x110000 - 0x7fffffff) can also be represented in UTF-9 by obvious extension, but this is not discussed further as these codepoints have been removed from [ISO-10646] by ISO.

UTF-18 encodes [UNICODE] codepoints in the Basic Multilingual Plane (BMP, plane 0), Supplementary Multilingual Plane (SMP, plane 1), Supplementary Ideographic Plane (SIP, plane 2), and Supplementary Special-purpose Plane (SSP, plane 14) in a single 18-bit value. It does not encode planes 3 though 13, which are currently unused; nor planes 15 or 16, which are private spaces.

Normally, UTF-9 and UTF-18 should only be used in the context of 9 bit storage and transport. Although some protocols, e.g., [FTP], support transport of nonets, the current IETF protocol suite is quite deficient in this area. The IETF is urged to take action to improve IETF protocol support for nonets.

\section{UTF-9 Definition}

A UTF-9 stream represents [ISO-10646] codepoints using 9 bit nonets. The low order 8-bits of a nonet is an octet, and the high order bit indicates continuation. 
UTF-9 does not use surrogates; consequently a UTF-16 value must be transformed into the UCS-4 equivalent, and U+D800 - U+DBFF are never transmitted in UTF-9.

Octets of the [UNICODE] codepoint value are then copied into successive UTF-9 nonets, starting with the most-significant non-zero octet. All but the least significant octet have the continuation bit set in the associated nonet.

Examples:

$\begin{array}{clll}\text { Character } & \text { Name } & \text { UTF-9 (in octal) } \\ ------- & ---- & ------------ \\ \text { U+0041 } & \text { LATIN CAPITAL LETTER A } & 101 \\ \text { U+00C0 } & \text { LATIN CAPITAL LETTER A WITH GRAVE } & 300 \\ \text { U+0391 } & \text { GREEK CAPITAL LETTER ALPHA } & 403221 \\ \text { U+611B } & \text { <CJK ideograph meaning "love"> } & 541 & 33 \\ \text { U+10330 } & \text { GOTHIC LETTER AHSA } & 40140360 \\ \text { U+E0041 } & \text { TAG LATIN CAPITAL LETTER A } & 416400101 \\ \text { U+10FFFD } & \text { <Plane 16 Private Use, Last> } & 420777375 \\ \text { 0x345ecflb } & \text { (UCS-4 value not in [UNICODE]) } & 46453671733\end{array}$

4. UTF-18 Definition

A UTF-18 stream represents [ISO-10646] codepoints using a pair of 9 bit nonets to form an 18-bit value.

UTF-18 does not use surrogates; consequently a UTF-16 value must be transformed into the UCS-4 equivalent, and U+D800 - U+DBFF are never transmitted in UTF-18.

[UNICODE] codepoint values in the range U+0000 - U+2FFFF are copied as the same value into a UTF-18 value. [UNICODE] codepoint values in the range U+E0000 - U+EFFFF are copied as values 0x30000 - 0x3ffff; that is, these values are shifted by $0 x 70000$. Other codepoint values can not be represented in UTF-18.

Examples:

$\begin{array}{lll}\text { Character } & \text { Name } & \text { UTF-18 (in octal) } \\ ------ & --- & ----------- \\ \text { U+0041 } & \text { LATIN CAPITAL LETTER A } & 000101 \\ \text { U+00C0 } & \text { LATIN CAPITAL LETTER A WITH GRAVE } & 000300 \\ \text { U+0391 } & \text { GREEK CAPITAL LETTER ALPHA } & 001621 \\ \text { U+611B } & \text { <CJK ideograph meaning "love"> } & 060433 \\ \text { U+10330 } & \text { GOTHIC LETTER AHSA } & 201460 \\ \text { U+E0041 } & \text { TAG LATIN CAPITAL LETTER A } & 600101\end{array}$




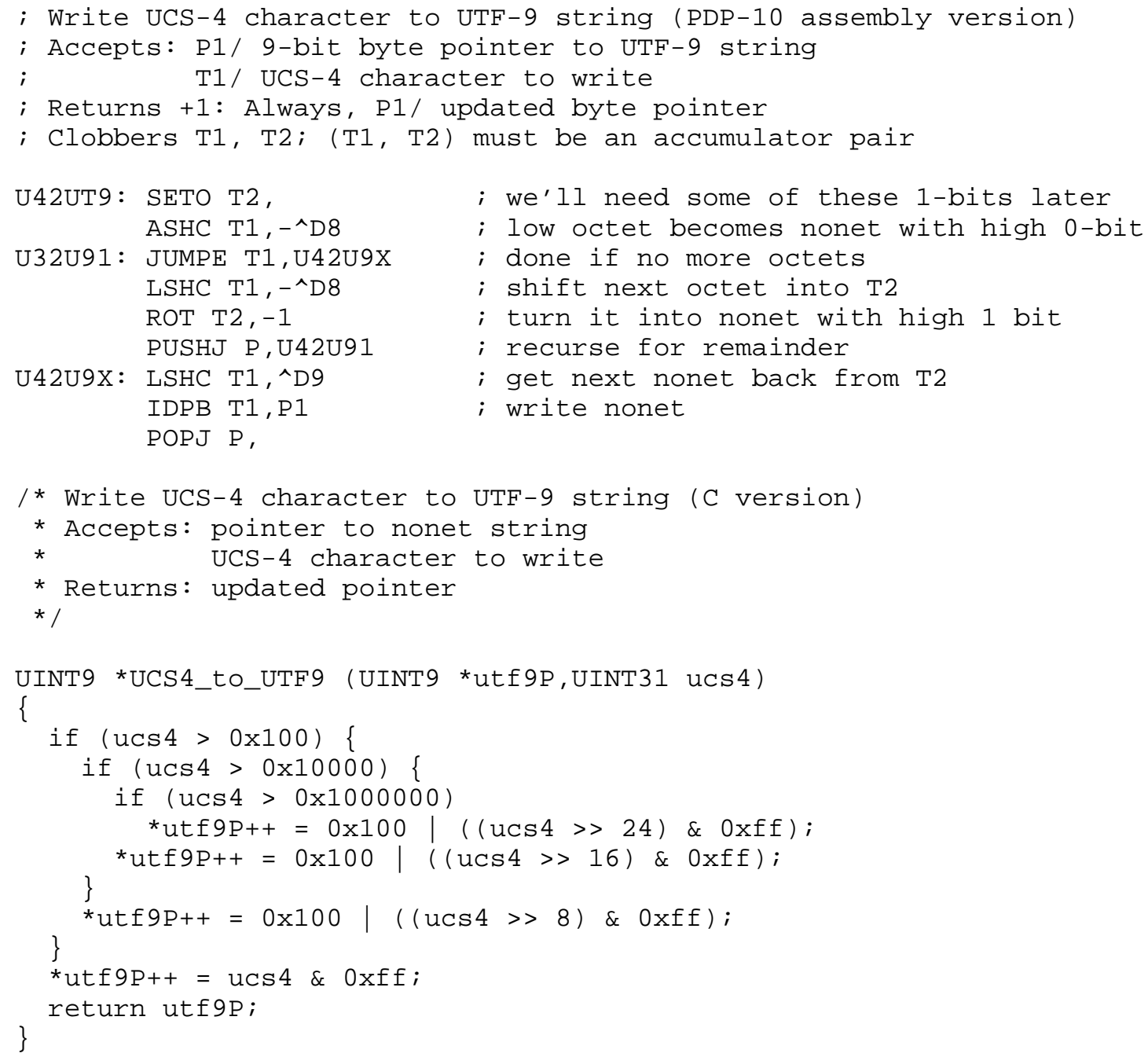

6. Implementation Experience

As the sample routines demonstrate, it is quite simple to implement UTF-9 and UTF-18 on a nonet-based architecture. More sophisticated routines can be found in ftp://panda.com/tops-20/utools.mac.txt or from lingling.panda.com via the file <UTF9>UTOOLS.MAC via ANONYMOUS [FTP]. 
We are now in the process of implementing support for nonet-based text files and automated transformation between septet, octet, and nonet textual data.

\section{References}

7.1. Normative References

[FTP] Postel, J. and J. Reynolds, "File Transfer Protocol", STD 9, RFC 959, October 1985.

[IAB-CHARACTER] Weider, C., Preston, C., Simonsen, K., Alvestrand, H., Atkinson, R., Crispin, M., and P. Svanberg, "The Report of the IAB Character Set Workshop held 29 February - 1 March, 1996", RFC 2130, April 1997.

[ISO-10646] International Organization for Standardization, "Information Technology - Universal Multiple-octet coded Character Set (UCS)", ISO/IEC Standard 10646, comprised of ISO/IEC 10646-1:2000, "Information technology - Universal Multiple-Octet Coded Character Set (UCS) - Part 1: Architecture and Basic Multilingual Plane", ISO/IEC 10646-2:2001, "Information technology - Universal Multiple-Octet Coded Character set (UCS) - Part 2: Supplementary Planes" and ISO/IEC 10646-1:2000/Amd 1:2002, "Mathematical symbols and other characters".

[KEYWORDS] Bradner, S., "Key words for use in RFCs to Indicate Requirement Levels", BCP 14, RFC 2119, March 1997.

[UNICODE] The Unicode Consortium, "The Unicode Standard Version 3.2", defined by The Unicode Standard, Version 3.0 (Reading, MA, Addison-Wesley, 2000. ISBN 0-201-61633-5), as amended by the Unicode Standard Annex \#27: Unicode 3.1 and by the Unicode Standard Annex \#28: Unicode 3.2, March 2002.

\subsection{Informative References}

[US-ASCII] American National Standards Institute, "Coded Character set - 7-bit American Standard Code for Information Interchange", ANSI X3.4, 1986.

[UTF-16] Hoffman, P. and F. Yergeau, "UTF-16, an encoding of ISO 10646", RFC 2781, February 2000. 
[UTF-7] Goldsmith, D. and M. Davis, "UTF-7 A Mail-Safe Transformation Format of Unicode", RFC 2152, May 1997 .

[UTF-8] Sollins, K., "Architectural Principles of Uniform Resource Name Resolution", RFC 2276, January 1998.

8. Security Considerations

As with UTF-8, UTF-9 can represent codepoints that are not in [UNICODE]. Applications should validate UTF-9 strings to ensure that all codepoints do not exceed the [UNICODE] maximum of U+10FFFF.

The sample routines in this document are for example purposes, and make no attempt to validate their arguments, e.g., test for overflow ([UNICODE] values great than 0xloffff) or codepoints used for surrogates. Besides resulting in invalid data, this can also create covert channels.

9. IANA Considerations

The IANA shall reserve the charset names "UTF-9" and "UTF-18" for future assignment.

Author's Address

Mark R. Crispin

Panda Programming

6158 NE Lariat Loop

Bainbridge Island, WA 98110-2098

Phone: (206) 842-2385

EMail: UTF9@Lingling.Panda.COM 
Full Copyright statement

Copyright (C) The Internet Society (2005).

This document is subject to the rights, licenses and restrictions contained in BCP 78 and at wWw.rfC-editor.org/copyright.html, and except as set forth therein, the authors retain all their rights.

This document and the information contained herein are provided on an "AS IS" basis and THE CONTRIBUTOR, THE ORGANIZATION HE/SHE REPRESENTS OR IS SPONSORED BY (IF ANY), THE INTERNET SOCIETY AND THE INTERNET ENGINEERING TASK FORCE DISCLAIM ALL WARRANTIES, EXPRESS OR IMPLIED, INCLUDING BUT NOT LIMITED TO ANY WARRANTY THAT THE USE OF THE INFORMATION HEREIN WILL NOT INFRINGE ANY RIGHTS OR ANY IMPLIED WARRANTIES OF MERCHANTABILITY OR FITNESS FOR A PARTICULAR PURPOSE.

Intellectual Property

The IETF takes no position regarding the validity or scope of any Intellectual Property Rights or other rights that might be claimed to pertain to the implementation or use of the technology described in this document or the extent to which any license under such rights might or might not be available; nor does it represent that it has made any independent effort to identify any such rights. Information on the procedures with respect to rights in RFC documents can be found in BCP 78 and BCP 79 .

Copies of IPR disclosures made to the IETF Secretariat and any assurances of licenses to be made available, or the result of an attempt made to obtain a general license or permission for the use of such proprietary rights by implementers or users of this specification can be obtained from the IETF on-line IPR repository at http://www.ietf.org/ipr.

The IETF invites any interested party to bring to its attention any copyrights, patents or patent applications, or other proprietary rights that may cover technology that may be required to implement this standard. Please address the information to the IETF at ietfipreietf.org.

Acknowledgement

Funding for the RFC Editor function is currently provided by the Internet Society. 